\title{
Comprensión Lectora y Cálculo Matemático: El Rol de la Memoria de Trabajo en Niños de Edad Escolar
}

\section{Reading Comprehension and Mathematical Computation: The Role of Working Memory in School-Age Children}

\author{
Santiago Vernucci, Lorena Canet-Juric, María Laura Andrés y Débora Inés Burin \\ Consejo Nacional de Investigaciones Científicas y Técnicas
}

\begin{abstract}
El objetivo del estudio fue analizar la capacidad explicativa de los componentes de la memoria de trabajo (MT) en las habilidades académicas de comprensión lectora (CL) y cálculo matemático (CM) en niños de edad escolar. La muestra estuvo compuesta por 84 niños argentinos de ambos sexos, de 9 a 11 años de edad, seleccionados mediante un muestreo no probabilístico intencional. La MT se evaluó mediante la batería informatizada de Tareas de Autorregulación Cognitiva (TAC), la CL, con la prueba de screening del Test Leer para Comprender y el CM, con el sub-test Cálculo Matemático del WRAT-3. Se realizaron análisis de regresión lineal múltiple y se calculó el porcentaje único de varianza explicada por cada componente. Los resultados mostraron que los componentes de almacenamiento verbal y ejecutivo explicaron la CL, mientras que el almacenamiento verbal, visoespacial y el componente ejecutivo explicaron el CM. Estos resultados son de interés para profundizar en el conocimiento del rol de la MT en la CL y el CM en niños del medio local.
\end{abstract}

Palabras clave: memoria de trabajo, comprensión lectora, cálculo matemático, niños

\begin{abstract}
The aim of the study was to analyze the explanatory power of the components of working memory (WM) with respect to reading comprehension (RC) and mathematical computation (MC) skills in school-age children. The sample was obtained through a purposive non probability procedure and consisted of 84 Argentinean children of both sexes, aged 9-11 years. WM was assessed with the computer-based Tareas de Autorregulación Cognitiva [Cognitive Self-Regulation Tasks] (TAC) battery, RC with the screening task from the Leer para Comprender [Reading to Understand] Test, and MC with the Mathematical Computation sub-test from the WRAT-3. Multiple linear regression analyses were performed and the unique percentage of variance explained by each component was calculated. Results showed that verbal storage and the executive component explained RC, while both verbal and visuo-spatial storage and the executive component explained MC. These results are important for gaining in-depth knowledge about the role of $\mathrm{WM}$ in $\mathrm{RC}$ and $\mathrm{MC}$ in local children.
\end{abstract}

Keywords: working memory, reading comprehension, mathematical computation, children

Las habilidades académicas en los niños son un componente significativo tanto de su desarrollo social y cognitivo como de su funcionamiento adaptativo en el entorno. Aquellos que exhiben dificultades académicas están en mayor riesgo de desarrollar trastornos emocionales y comportamentales (Bennett, Brown, Boyle, Racine \& Offord, 2003) y de ver comprometida su inserción laboral futura (Alonso \& Mateos, 1985; Parsons \& Bynner, 2005). Dos de las principales habilidades académicas son la comprensión lectora (CL) y el cálculo matemático (CM; Pickering, 2006).

La CL es un proceso complejo que implica la orquestación de diversas habilidades y procesos cognitivos, que van desde la decodificación y reconocimiento de palabras hasta procesos de alto nivel, como la integración del significado de las distintas partes del material leído, con el objetivo de construir un modelo mental coherente del texto (Canet-Juric, Burin, Andrés \& Urquijo, 2013; Oakhill, Cain \& Elbro, 2015). Constituye el medio básico por el que se adquiere información en nuestra sociedad. La comprensión de textos implica la formación de una representación del sentido de lo leído, que toma la forma de un modelo

Santiago Vernucci, Lorena Canet-Juric y María Laura Andrés, Instituto de Psicología Básica, Aplicada y Tecnología (IPSIBAT), Universidad Nacional de Mar del Plata, Consejo Nacional de Investigaciones Científicas y Técnicas (CONICET), Mar del Plata, Argentina; Débora Inés Burin, Facultad de Psicología, Universidad de Buenos Aires, CONICET, Buenos Aires, Argentina.

Este artículo ha sido elaborado en el marco de una beca interna doctoral otorgada por el CONICET al primer autor y de proyectos financiados por la Agencia Nacional de Promoción Científica y Tecnológica y el CONICET.

La correspondencia relativa a este artículo debe ser dirigida a Santiago Vernucci, Instituto de Psicología Básica, Aplicada y Tecnología, Facultad de Psicología, Universidad Nacional de Mar del Plata, Funes 3250, Cuerpo V, Nivel III, Mar del Plata, Argentina. E-mail: santiagovernucci@conicet.gov.ar 
mental o de situación, integrado y coherente. Para lograrlo es necesario que operen los procesos de integración e inferencia. La integración entre palabras y oraciones es necesaria para poder establecer la coherencia local, y las inferencias acerca de diferentes eventos, acciones y estados son necesarias para que el texto forme una totalidad coherente. Además, estos procesos requieren que la información relevante, tanto del texto como del conocimiento del mundo que tiene el lector, estén disponibles y en un estado accesible (Cain, Oakhill \& Bryant, 2004).

Las matemáticas suponen un concepto amplio, ya que implican la evaluación de propiedades y relaciones de cantidad expresadas en números o símbolos. Involucran una variedad de capacidades complejas que posibilitan el manejo de operaciones y contenido conceptual diverso (e.g., aritmética, álgebra, geometría). Es posible considerar diferentes áreas dentro de las matemáticas: conocimiento numérico básico (cardinalidad, ordinalidad, conteo, conocimiento de dígitos arábigos y estimación numérica), cálculo con números enteros (de uno y múltiples dígitos), fracciones, geometría, álgebra y resolución de problemas verbales (Peng, Namkung, Barnes \& Sun, 2016; Raghubar, Barnes \& Hecht, 2010). Si se considera particularmente al CM, es una habilidad que implica la capacidad de ejecutar suma, resta, multiplicación y división tanto de uno como de múltiples dígitos, lo que supone la resolución de problemas aritméticos simples, como " $3+4$ ", y problemas más complejos, como divisiones y fracciones (Hecht, Torgesen, Wagner \& Rashotte, 2001).

Estas dos habilidades no solo son importantes para un desempeño exitoso en la escuela, sino que son cruciales para el aprendizaje en general, el éxito en la educación y en múltiples situaciones cotidianas a lo largo de la vida (Bull \& Lee, 2014; Oakhill et al., 2015), por lo que cobra valor conocer los factores que pueden influir en su adquisición y desarrollo. Uno de estos factores es la memoria de trabajo (MT; Alloway \& Copello, 2013; Alloway \& Passolunghi, 2011; Bull \& Lee, 2014; St Clair-Thompson \& Gathercole, 2006; Swanson \& Alloway, 2012), que puede definirse como un sistema complejo de capacidad limitada encargado de almacenar y procesar información de manera simultánea, lo que posibilita la ejecución de un amplio rango de actividades cognitivas (Baddeley, 2012; Baddeley \& Logie, 1999; Diamond, 2013). Por ello, está implicada en situaciones tan diversas como la capacidad de otorgarle sentido a información de carácter lingüístico, la regulación de las emociones y del comportamiento, la comprensión de cualquier situación que se desarrolle gradualmente en el tiempo, el reordenamiento y manipulación mental de información, el establecimiento de relaciones de causa-efecto y la posibilidad de relacionar piezas de información para lograr inferir principios generales o establecer nuevas relaciones entre ideas previas (Diamond, 2012; Hofmann, Schmeichel \& Baddeley, 2012).

Si bien existen conceptualizaciones diversas de la MT (véase Miyake \& Shah, 1999), los modelos de MT que han recibido mayor apoyo en distintas investigaciones la presentan como un sistema compuesto por componentes de almacenamiento de dominio específico, encargados de la retención a corto plazo de diversos tipos de información (e.g., verbal y visoespacial) y un componente de domino general, encargado del procesamiento, manipulación, actualización e integración de la información, así como del control de recursos atencionales (Baddeley, 1996, 2003a, 2012; Engle, Kane \& Tuholski, 1999; Kane et al., 2004). En línea con lo anterior, uno de los modelos más influyentes de MT es el que la caracteriza como un sistema formado por diversos componentes especializados, que funcionan en conjunto para posibilitar el almacenamiento y procesamiento de la información. Desde esta concepción, la MT puede ser dividida en tres componentes principales. El bucle fonológico es el componente encargado de almacenar temporariamente información verbal, típicamente por unos pocos segundos, y de la repetición (rehearsal) de esa información de manera verbal o sub vocal, para evitar su decaimiento. La agenda visoespacial es el componente encargado de almacenar temporariamente información de tipo visual y espacial. Estos dos componentes son específicos según la modalidad sensorial de la información y cuentan con una capacidad de almacenamiento limitada. El componente restante es el ejecutivo central, que puede caracterizarse como un sistema encargado de la coordinación de los dos almacenes y del control atencional, manteniendo y redirigiendo los procesos de focalización y cambio atencional. A diferencia de los componentes encargados del almacenamiento, el ejecutivo central no cuenta con especificidad en cuanto a la modalidad de la información sensorial con la que opera, por lo que es un componente de dominio general (Baddeley, 1992, 1996, 2003a, 2010). Es importante destacar que este modelo de MT ha evidenciado un adecuado ajuste en población de edad escolar: desde los cuatro años hasta entrada la adolescencia, la MT presenta una organización estructural consistente con este modelo y, además, su capacidad se incrementa de forma sostenida a lo largo de dicho período (Alloway, Gathercole \& Pickering, 2006; Gathercole, Pickering, Ambridge \& Wearing, 2004). 
En cuanto a la relación entre la MT y las diferentes habilidades académicas, el rol que juega la MT es clave, ya que la evidencia indica que constituye un importante predictor del desempeño en lectura y matemáticas (Gathercole, Alloway, Willis \& Adams, 2006; Gathercole, Pickering, Knight \& Stegmann, 2004; St Clair-Thompson \& Gathercole, 2006; Swanson \& Alloway, 2012) y que, incluso, cuando se la evalúa a los cinco años de edad, constituye el mejor predictor del desempeño académico seis años después, aún si se considera el coeficiente intelectual (Alloway \& Alloway, 2010).

Si se analiza la relación entre la MT y la CL, la evidencia indica que la MT posibilita al lector mantener de manera activa en su foco atencional las representaciones más relevantes del texto, a medida que lo va leyendo (Oakhill et al., 2015; Richard's, Canet-Juric, Introzzi \& Urquijo, 2014). De hecho, la MT opera almacenando las palabras u oraciones que fueron recientemente leídas, permitiendo su integración para establecer coherencia, y, a su vez, mantiene la información recuperada de la memoria a largo plazo para facilitar su integración con el texto actualmente activo (Cain et al., 2004; Daneman \& Merikle, 1996). La MT tiene un rol activo en la comprensión de textos, tal como lo muestran los estudios empíricos (Swanson, 2003; Swanson, Zheng \& Jerman, 2009). La adecuada comprensión de un texto implica que el lector logre integrar el significado de aquello que lee. Para ello, las palabras y oraciones que son leídas deben mantenerse activas a medida que se avanza en la lectura y se integra el contenido leído previamente con el más reciente. Es decir, se necesita almacenamiento y procesamiento concurrente de la información, lo que supone la intervención de los procesos ejecutivos de la MT. De hecho, existe evidencia que muestra la implicación del componente ejecutivo en la CL (e.g., Cain et al., 2004; Swanson et al., 2009). En cuanto al bucle fonológico, su rol es clave en las habilidades lingüísticas de los niños, ya que sustenta la adquisición de vocabulario, apoyando la formación de representaciones fonológicas estables de nuevas palabras en la memoria a largo plazo. El bucle fonológico opera facilitando la adquisición del lenguaje, al brindar la posibilidad de representar temporalmente nuevas secuencias de fonemas, y permitiendo su aprendizaje mediante la repetición, lo que impacta en la comprensión de textos, ya que el grado de conocimiento semántico es un factor clave en dicha habilidad. Se ha mostrado que la posibilidad de retener la información verbal recientemente leída durante algunos segundos permite mantenerla activa para que puedan operar las diversas habilidades de comprensión (Baddeley, 2003a, 2003b; Cain et al., 2004; Engel de Abreu, Gathercole \& Martin, 2011). Finalmente, se ha mostrado que el componente de almacenamiento visoespacial disminuye su rol en procesos como la comprensión de textos a medida que los niños desarrollan la capacidad de recodificación fonológica de la información y prefieren, entonces, su almacenamiento en forma de representaciones verbales, aproximadamente a partir de los siete años de edad (Baddeley, 2003b; Pickering, 2001). En síntesis, la evidencia indica que la MT estaría implicada en la CL, principalmente a través del componente ejecutivo y de almacenamiento verbal, y que el componente visoespacial perdería importancia en los niños de mayor edad. Debe destacarse que generalmente no se aborda el análisis de la contribución conjunta de los componentes de MT a la CL. De modo que analizar la contribución de los componentes de la MT a la CL representaría un aporte a los estudios ya realizados, al permitir conocer la contribución relativa de cada factor cuando se controla el peso de los restantes, así como la contribución explicativa única de cada uno de ellos.

Si el foco se pone en la relación entre la MT y el CM, la evidencia indica que la MT está implicada en el $\mathrm{CM}$ cuando es necesario retener información parcial y procesar de manera concurrente nueva información para llegar a una solución (Bull \& Lee, 2014; Raghubar et al., 2010). Las operaciones de cálculo con números enteros requieren regular y mantener combinaciones aritméticas que pueden estar derivadas tanto de su recuperación desde la memoria a largo plazo como del conteo, mientras que simultáneamente se atiende a las demandas de reagrupación y a los valores posicionales. En ese sentido, la MT contribuye al $\mathrm{CM}$, porque requiere del almacenamiento y procesamiento de información de manera simultánea (Peng et al., 2016). Si se considera a las matemáticas en general, existe evidencia de una relación significativa y moderada con la MT, y se ha reportado un efecto moderador del tipo de habilidad matemática (Friso-van den Bos, van der Ven, Kroesbergen \& van Luit, 2013; Peng et al., 2016; Raghubar et al., 2010). Así, la resolución de problemas verbales y el CM muestran relaciones más fuertes con la MT que otras habilidades, como la geometría (Peng et al., 2016). Un factor que podría resultar de peso en el desempeño en el CM es la edad del niño, dado que a medida que el niño crece y avanza en su escolaridad es esperable que aumente su nivel de habilidad y experticia en diferentes dominios de las matemáticas (Raghubar et al., 2010).

Se han indicado distintas funciones para cada componente de la MT en el CM. Se ha mostrado que el ejecutivo central opera como un componente de dominio general, que se relaciona de manera moderada con el CM (Peng et al., 2016), y que esta relación tiene lugar cuando es necesario retener y procesar de manera 
concurrente los resultados de las diferentes operaciones (Alloway \& Passolunghi, 2011; Friso-van den Bos et al., 2013). Numerosos estudios han demostrado que la habilidad para procesar fonológicamente la información se asocia a la capacidad en matemáticas; específicamente, la habilidad para manipular la estructura fonológica del lenguaje tiene un rol importante en el cálculo aritmético, ya que este componente permite mantener en un estado activo la información numérica relevante para la resolución del problema (Alloway \& Passolunghi, 2011; Gathercole et al., 2006; Geary, Hoard, Nugent \& Byrd-Craven, 2008; Hecht et al., 2001). Finalmente, también existe evidencia de la contribución de la agenda visoespacial al CM (Alloway \& Passolunghi, 2011; Friso-van den Bos et al., 2013; Hecht et al., 2001) y se ha sugerido que posiblemente opera como una "pizarra" mental, permitiendo la representación numérica, especialmente cuando los problemas son presentados en un formato visual (Geary et al., 2008; McLean \& Hitch, 1999; Trbovich \& LeFevre, 2003). En síntesis, la evidencia indica que la MT estaría implicada en el CM a través de cada uno de sus componentes. Sin embargo, los estudios generalmente no abordan la contribución conjunta de cada uno de los componentes de la MT al CM. Analizar la contribución de los componentes aportaría nueva evidencia a la ya revelada, al mostrar el peso relativo de cada factor cuando se controla la contribución de los restantes, así como la contribución explicativa única de cada uno de ellos.

La capacidad de MT de los niños puede afectar su desempeño y aprendizaje. Una baja capacidad de MT limita la posibilidad de adquirir y consolidar estas habilidades, ya que puede actuar como un "cuello de botella" para el aprendizaje (Alloway, 2006; Gathercole, 2004), por lo que el estudio detallado de las relaciones entre la MT y las distintas habilidades académicas resulta de sumo valor. La evidencia revisada indica que la MT está implicada en la CL, a través del componente ejecutivo y del almacenamiento verbal principalmente, y en el CM, a través de todos los componentes. No obstante, la investigación se ha centrado mayormente en el rol de alguno de los componentes por sobre otros (e.g., el componente ejecutivo; Peng et al., 2016; Swanson et al., 2009). Por lo anterior, resulta importante considerar los componentes de la MT de manera conjunta para analizar su contribución explicativa en las habilidades académicas de CL y CM, lo que permitiría conocer el peso de cada uno cuando se controla el de los restantes factores, así como la contribución explicativa única de cada factor. Adicionalmente, evaluar conjuntamente MT, CL y CM, puede permitir indagar si la MT está implicada de manera diferencial en tales habilidades académicas.

Si bien se ha señalado que la MT predice el desempeño académico y se ha reportado evidencia de la implicación de la MT en las habilidades de CL y CM, la cantidad de estudios con niños del medio local es comparativamente menor a la registrada en otros contextos, principalmente Europa y Estados Unidos (Friso-van den Bos et al., 2013; Swanson et al., 2009). Esto limita la posibilidad de generalizar los resultados al contexto argentino, de modo que resulta de valor ahondar en el estudio de la relación entre la MT y las habilidades académicas de CL y CM en esta población.

El objetivo de este trabajo fue analizar la capacidad explicativa conjunta y única de los componentes del sistema de MT en las habilidades académicas de CL y CM en niños argentinos escolarizados de 9 a 11 años de edad, así como conocer el peso diferencial de la MT en cada una de estas habilidades académicas.

Se hipotetizó que (a) una porción significativa de la varianza de la CL es explicada por el componente ejecutivo y por el de almacenamiento verbal de la MT y (b) una porción significativa de la varianza del CM es explicada por los tres componentes de la MT.

\section{Método}

\section{Diseño}

Se utilizó un diseño transversal correlacional.

\section{Participantes}

Mediante un muestreo intencional no probabilístico, se seleccionaron 84 niños (48 mujeres y 36 varones) de entre 9 y 11 años de edad ( $M=10$ años y 4 meses; $D E=6$ meses) que se encontraban cursando cuarto $(n=54)$ y quinto $(n=30)$ año de educación primaria, provenientes de dos instituciones educativas de gestión privada de la ciudad de Mar del Plata, Argentina. El 70\% de los participantes provenía de familias de estatus social medio. El resto se distribuía en familias de estatus social bajo (22\%) y alto (8\%). Para calcular el estatus social se utilizó el Índice de Status Social de Hollingshead (2011). 


\section{Instrumentos}

Memoria de trabajo. Se utilizaron las tareas informatizadas de la Batería de Tareas de Autorregulación Cognitiva (TAC; Canet-Juric, Introzzi \& Burin, 2015; Introzzi, Canet-Juric, Comesaña, Andrés \& Richard's, 2013), las cuales permiten la evaluación independiente de los diferentes componentes del sistema de MT y que están basadas en el paradigma dual (Hale, Bronik \& Fry, 1997). Las tareas son de dos tipos: simples y duales. Las tareas simples consisten en la retención a corto plazo de distintos ítems. Las tareas duales pueden caracterizarse como de amplitud compleja, ya que requieren la ejecución simultánea de dos tareas: una tarea primaria simple como la anterior y una tarea secundaria simultánea de interferencia, que tiene como objetivo interrumpir cualquier tipo de estrategia que pudiera facilitar el mantenimiento de la información de la tarea primaria. Las tareas simples permiten evaluar el componente de almacenamiento (verbal y visoespacial), mientras que las tareas con interferencia, el componente ejecutivo de la MT (Conway et al., 2005; Hale, Myerson, Emery, Lawrence \& DuFault, 2007; Schmiedek, Hildebrandt, Lövdén, Wilhelm \& Lindenberger, 2009).

Todas las tareas comienzan con una fase de práctica, en la que el administrador se asegura que el participante ha comprendido la consigna. Cada tarea se inicia con la presentación de dos ítems consecutivos. Si el participante da una respuesta correcta, se agrega un ítem adicional en la próxima secuencia. Si el participante da una respuesta incorrecta, se presenta nuevamente una secuencia con la misma cantidad de ítems y, si la respuesta fuera nuevamente incorrecta, finaliza la prueba. La cantidad máxima de ítems que se presentan son 9. Se obtiene una estimación de amplitud para cada tarea, que corresponde a la máxima cantidad de ítems recordados correctamente. Las tareas de MT que integran la Batería TAC han registrado evidencias de validez interna, mostrando mayor amplitud en tareas simples que con interferencia, tanto en el dominio verbal como visoespacial; además, evidencias de validez externa, en tanto no se registraron diferencias significativas entre los puntajes medios obtenidos en cada tarea y los del estudio de Hale et al. (1997), en el que están basadas las tareas (Canet-Juric et al., 2015).

Componentes de almacenamiento. Se utilizó la tarea verbal simple y la tarea visoespacial simple. La tarea verbal simple consiste en presentar en la pantalla una serie de dígitos de manera serial, seguidos de una señal sonora de recordar. Luego, el participante debe seleccionar con el mouse en una grilla los dígitos que le fueron mostrados en el mismo orden en que se presentaron. La tarea visoespacial simple le presenta al niño en la pantalla una matriz de $4 \times 4$ celdas, dentro de las cuales aparecen cruces de manera serial, seguidas de una señal sonora de recordar. Luego, el participante debe indicar con el mouse en una matriz vacía el lugar donde estaban las cruces en el mismo orden en que se presentaron. Se consideró la variable almacenamiento verbal como la amplitud de la tarea verbal simple y la variable almacenamiento visoespacial como la amplitud de la tarea visoespacial simple; en ambos casos se usaron puntajes brutos de amplitud, los cuales van de un mínimo de 2 a un máximo posible de 9 . Se obtuvo una consistencia interna, evaluada mediante alfa de Cronbach, de 0,55 para la tarea verbal y 0,62 para la tarea visoespacial.

Componente ejecutivo. Se utilizó la tarea verbal con interferencia y la tarea visoespacial con interferencia. La tarea verbal con interferencia presenta en la pantalla una serie de dígitos coloreados (rojos, azules o verdes) de uno en uno, seguidos de una señal sonora de recordar. De manera simultánea, el participante debe decir en voz alta, inmediatamente desaparece de la pantalla cada dígito, el color en el que fue presentado. Una vez que termina la presentación, el participante debe seleccionar con el mouse en una grilla los dígitos que le fueron mostrados en el mismo orden en que se presentaron. La tarea visoespacial con interferencia presenta en la pantalla una matriz de $4 \times 4$ celdas, dentro de las cuales aparecen cruces coloreadas (rojos, azules o verdes) de una en una, seguidas de una señal sonora de recordar. De manera simultánea, luego de cada ítem el participante debe indicar en una paleta de colores que se ubica a la derecha de la matriz el color en que se presentó el ítem. Una vez que termina la presentación, el participante debe indicar con el mouse en una matriz vacía el lugar donde estaban las cruces en el mismo orden en que se presentaron. Se consideró la variable procesamiento ejecutivo mediante un índice calculado por medio de promediar las puntuaciones brutas de amplitud, obtenidas en las tareas con interferencia (verbal y visoespacial). Los puntajes de amplitud de cada tarea van de un mínimo de 2 a un máximo posible de 9, lo mismo que del índice del componente ejecutivo. Los valores de consistencia interna, evaluada mediante alfa de Cronbach, fueron 0,50 para la tarea verbal y 0,60 para la tarea visoespacial. 
Comprensión lectora. Se utilizó la prueba de screening para niños de 9 a 11 años de edad del Test Leer para Comprender (TLC; Abusamra, Ferreres, Reiter, De Beni \& Cornoldi, 2010). El TLC es una prueba que evalúa exhaustivamente las habilidades de comprensión de textos y está validada para su utilización con niños argentinos de 9 a 12 años de edad. La prueba de screening permite evaluar diferentes áreas del proceso de CL, obteniendo una valoración general del mismo. La prueba consiste en la presentación de dos textos: uno narrativo y uno expositivo, sobre los que se realizan 10 preguntas de opción múltiple a cada uno. Cada respuesta contempla cuatro opciones, de las cuales solo una es correcta, y las tres restantes son distractores con distinto grado de proximidad semántica respecto de la correcta. Se asigna 1 punto por cada respuesta correcta y 0 por respuesta incorrecta, falta de respuesta o selección de más de una opción. La puntuación en cada texto varía desde un mínimo de 0 a un máximo posible de 10 puntos. Los valores de consistencia interna reportados para la muestra normativa resultan adecuados, siendo de $\quad=0,69$ tanto para el texto expositivo como para el narrativo (Abusamra et al., 2010). En esta muestra se obtuvo $=0,77$ para el texto narrativo $\mathrm{y}=0,74$ para el expositivo. El tiempo medio de administración del texto narrativo es de 8,46 minutos y del texto expositivo, 10,21 minutos. En el presente estudio se calculó un índice de CL, constituido por el promedio de los puntajes brutos de ambos textos, siendo 0 el puntaje mínimo posible y 10 el máximo.

Cálculo matemático. Se utilizó el sub-test Cálculo Matemático de la Prueba de Logro de Amplio Rango WRAT-3 (Wilkinson, 1993). Este sub-test permite evaluar las habilidades básicas de conteo, lectura de símbolos numéricos, resolución de problemas orales y desempeño en cálculos escritos. Esta prueba está diseñada para las edades comprendidas desde los 5 hasta los 74 años. A partir de los 8 años de edad se administra el apartado Aritmética Escrita, que consiste en 40 ejercicios de cálculos aritméticos de dificultad creciente, comenzando por problemas de adición de un dígito hasta problemas de álgebra que contienen varias variables. Se computa 1 punto por cada ejercicio correctamente resuelto y 0 punto por ejercicio incorrecto o no respondido. El tiempo de administración de la prueba no debe exceder los 15 minutos y la prueba puede interrumpirse si el participante indica que ya ha completado todos los ítems que es capaz de resolver. Dada la edad de los participantes de la muestra, en el presente trabajo se administró el apartado Aritmética Escrita. La variable CM consistió en la suma de los ejercicios resueltos correctamente, siendo 0 el puntaje bruto mínimo posible y 40 el máximo. Las propiedades psicométricas de consistencia interna y validez de la prueba han mostrado ser adecuadas para los diferentes grupos de edad (Snelbaker, Wilkinson, Robertson \& Glutting, 2001). Se obtuvo = 0,76 para la muestra evaluada.

\section{Procedimiento}

Las instituciones fueron invitadas a participar del estudio, en el marco de un convenio preexistente con el grupo de investigación. Se llevaron a cabo reuniones con cada equipo directivo para informar sobre el tipo de evaluaciones que se iban a llevar a cabo y el tipo de información que se esperaba obtener y coordinar las franjas horarias y espacio físico para el desarrollo de las actividades. Una vez convenido esto, para la selección de participantes se envió a los padres un documento informativo acerca del estudio, junto con el formulario de consentimiento informado. Solo los niños cuyos padres y/o tutores dieron el consentimiento fueron incluidos en la muestra; asimismo, se contó con el asentimiento del niño al momento de realizar las evaluaciones. La administración de los instrumentos tuvo lugar dentro del establecimiento educativo al que concurría cada participante, durante el horario escolar habitual. Las pruebas fueron administradas por un equipo conformado por los autores del trabajo, así como por evaluadores especialmente entrenados para la administración de las tareas, los cuales cuentan con experiencia previa en evaluaciones en contexto escolar y están familiarizados con las pruebas administradas.

Se desarrollaron dos sesiones de evaluación: en la primera se administraron individualmente las tareas de MT de la Batería TAC; en la segunda, se administraron grupalmente las pruebas de CL y CM. Ambas sesiones tuvieron lugar en salones ubicados en áreas tranquilas de cada escuela, acordados con los directivos y acondicionados a los fines de la administración de los instrumentos.

Para la implementación de esta investigación se tomaron los lineamientos éticos que comprenden las actividades destinadas a obtener conocimientos sobre procesos psicológicos en seres humanos, recomendados por la American Psychological Association (2010) y los lineamientos dados por CONICET (2006) para el comportamiento ético en las ciencias sociales y humanidades. 


\section{Análisis de Datos}

En primer lugar, se calcularon los índices del componente ejecutivo y de CL. El índice del componente ejecutivo se obtuvo promediando las amplitudes obtenidas en las tareas con interferencia (verbal y visoespacial). El índice de CL se obtuvo promediando las puntuaciones obtenidas en los dos textos (narrativo y expositivo) de la prueba. En segundo lugar, se realizaron análisis descriptivos de las variables bajo estudio, obteniendo los valores medios, desvíos estándar, puntuación mínima y máxima e índices de asimetría y curtosis de cada variable. En tercer lugar, se realizaron análisis de regresión lineal múltiple para conocer el peso relativo de cada componente de MT sobre las habilidades académicas. Se realizaron dos modelos, uno para CL y otro para CM. En ambos se introdujeron los distintos componentes junto con la edad de los participantes como variables independientes y la CL o el CM como variable dependiente. Para controlar posibles relaciones espurias entre los componentes de la MT con las habilidades académicas que se pudieran deber a las diferencias de edad de los participantes, se optó por incluir en los modelos la edad como variable independiente. En cuarto lugar, se analizó la contribución explicativa única de cada factor a las habilidades académicas. Para esto se realizaron análisis de porcentaje único de varianza explicada mediante modelos de regresión adicionales que poseían solo dos componentes, junto con la edad, como variables independientes y cuyos $R^{2}$ fueron restados de los $R^{2}$ de los modelos principales. Las diferencias entre ellos indican el porcentaje de varianza explicada única por cada uno de los componentes en cada una de las habilidades académicas.

\section{Resultados}

\section{Análisis Descriptivos}

Los estadísticos descriptivos de las puntuaciones objetivas en las diferentes tareas, así como del índice del componente ejecutivo de la MT, el índice de CL y la edad se presentan en la Tabla 1. En todos los casos los valores de asimetría y curtosis indicaron una distribución normal de los datos, lo que permite la utilización de técnicas paramétricas (Martín Martín, Cabero Morán \& de Paz Santana, 2008).

Tabla 1

Estadísticos Descriptivos Para las Variables Bajo Estudio

\begin{tabular}{lcccccr}
\hline \multicolumn{1}{c}{ Variable } & $M$ & $D E$ & Mín. & Máx. & \multicolumn{1}{c}{$A S$} & \multicolumn{1}{c}{$K S$} \\
\hline Tarea verbal simple & 4,95 & 0,93 & 2,00 & 7,00 & $-0,10$ & 0,62 \\
Tarea visoespacial simple & 4,84 & 1,38 & 2,00 & 8,00 & $-0,30$ & $-0,33$ \\
Tarea verbal con interferencia & 3,23 & 0,87 & 2,00 & 5,00 & 0,09 & $-0,78$ \\
Tarea visoespacial con interferencia & 3,44 & 1,05 & 2,00 & 5,00 & 0,13 & $-1,16$ \\
Índice componente ejecutivo (MT) & 3,33 & 0,73 & 2,00 & 4,50 & $-0,08$ & $-0,95$ \\
Texto expositivo (screening TLC) & 6,95 & 2,44 & 0,00 & 10,00 & $-0,96$ & 0,52 \\
Texto narrativo (screening TLC) & 6,41 & 2,68 & 0,00 & 10,00 & $-0,70$ & $-0,31$ \\
Índice comprensión lectora & 6,68 & 2,33 & 0,50 & 10,00 & $-0,68$ & $-0,43$ \\
Cálculo matemático (WRAT-3) & 12,81 & 3,35 & 2,00 & 19,00 & $-0,89$ & 1,07 \\
Edad & 10,31 & 0,55 & 9,33 & 11,50 & 0,17 & $-0,94$ \\
\hline
\end{tabular}

\section{Contribución Explicativa de los Diferentes Componentes de MT: Análisis de Regresión Lineal Múltiple}

Se realizaron dos modelos de regresión que incluyeron los componentes de almacenamiento verbal, almacenamiento visoespacial y ejecutivo de la MT, y la edad como variables independientes, junto con la CL o el CM como variables dependientes.

Los resultados del análisis de regresión del modelo de CL (ver Tabla 2) muestran que el modelo resulta significativo, explicando un 33\% de la varianza de esta habilidad académica, $F(4,74)=9,107, p<0,001$. En cuanto a la capacidad explicativa de cada variable controlando el peso de las restantes, se observa que el 
componente ejecutivo y el de almacenamiento verbal resultan predictores significativos de la CL, mientras que el almacenamiento visoespacial y la edad de los participantes no lo son.

En lo que respecta al CM, los resultados del análisis de regresión (ver Tabla 2) muestran que el modelo propuesto resulta significativo, explicando un $52,3 \%$ de la varianza de esta habilidad académica, $F(4,77)=21,212, p<0,001$. En cuanto a la capacidad explicativa de cada variable, se observa que los tres componentes de la MT resultan predictores significativos, mientras la edad de los participantes no realiza un aporte significativo. Finalmente, puede observarse que la MT explica una proporción de la varianza mayor del rendimiento en CM que en CL.

Tabla 2

Análisis de Regresión Lineal: Relación de los Componentes de la Memoria de Trabajo con la Comprensión Lectora y el Cálculo Matemático

\begin{tabular}{|c|c|c|c|c|c|c|c|c|c|c|}
\hline & \multicolumn{5}{|c|}{ Comprensión lectora } & \multicolumn{5}{|c|}{ Cálculo matemático } \\
\hline & $R^{2}$ & $g l$ & $\beta$ & $t$ & $p$ & $R^{2}$ & $g l$ & $\beta$ & $t$ & $p$ \\
\hline Modelo & 0,330 & 4,74 & & & & 0,523 & 4,77 & & & \\
\hline Componente ejecutivo & & & 0,29 & 2,55 & 0,013 & & & 0,20 & 2,15 & 0,034 \\
\hline Almacenamiento verbal & & & 0,31 & 2,89 & 0,005 & & & 0,48 & 5,47 & $<0,001$ \\
\hline Almacenamiento visoespacial & & & 0,14 & 1,25 & 0,216 & & & 0,25 & 2,72 & 0,008 \\
\hline Edad en meses & & & 0,02 & 0,17 & 0,868 & & & $-0,01$ & $-0,08$ & 0,935 \\
\hline
\end{tabular}

\section{Contribución Explicativa Única de Cada Componente: Análisis de Porcentaje Único de Varianza Explicada}

Para cada habilidad académica se realizaron tres modelos de regresión adicionales (seis en total): el primero con el componente ejecutivo y de almacenamiento verbal como variables independientes, el segundo con el componente ejecutivo y de almacenamiento visoespacial y el tercero con el componente de almacenamiento verbal y visoespacial como variables independientes, además de la edad. Los $R^{2}$ de cada uno de estos modelos adicionales fueron restados del $R^{2}$ de los modelos principales, obteniendo así el porcentaje de varianza explicada única por cada componente.

Los resultados (ver Tabla 3) muestran que para la habilidad académica de CL el componente ejecutivo posee un porcentaje de varianza explicativa única del 5,9\%, el almacenamiento verbal, del 7,6\% y el almacenamiento visoespacial, del 1,4\%. Para el CM, el componente ejecutivo de la MT posee un 2,9\% de la varianza explicativa única de esta habilidad, el almacenamiento verbal un $18,5 \%$ y el almacenamiento visoespacial un $4,6 \%$.

\section{Discusión}

El objetivo del presente estudio fue analizar la capacidad explicativa de los distintos componentes del sistema de MT en las habilidades de CL y CM en niños argentinos escolarizados de 9 a 11 años de edad. En función de la evidencia disponible, se hipotetizó que la CL sería explicada por el componente ejecutivo y por el de almacenamiento verbal de la MT, mientras que el CM sería explicado por los tres componentes de la MT. 
Tabla 3

Modelos de Regresión Adicionales Para Conocer la Varianza Explicativa Única de Cada Factor en Comprensión Lectora y Cálculo Matemático

\begin{tabular}{|c|c|c|c|c|c|c|c|c|c|c|c|c|}
\hline & \multicolumn{6}{|c|}{ Comprensión lectora } & \multicolumn{6}{|c|}{ Cálculo matemático } \\
\hline & \multicolumn{2}{|c|}{ (1) Visoesp. } & \multicolumn{2}{|c|}{ (2) Verbal } & \multicolumn{2}{|c|}{ (3) Ejecutivo } & \multicolumn{2}{|c|}{ (4) Visoesp. } & \multicolumn{2}{|c|}{ (5) Verbal } & \multicolumn{2}{|c|}{ (6) Ejecutivo } \\
\hline & $R^{2}$ & $\beta$ & $R^{2}$ & $\beta$ & $R^{2}$ & $\beta$ & $R^{2}$ & $\beta$ & $R^{2}$ & $\beta$ & $R^{2}$ & $\beta$ \\
\hline Modelo & 0,316 & & 0,254 & & 0,271 & & 0,477 & & 0,338 & & 0,494 & \\
\hline Ejecutivo & & $0,34^{* *}$ & & $0,38^{* * *}$ & & - & & $0,30 * * *$ & & $0,36^{* * *}$ & & \\
\hline Verbal & & $0,33^{* *}$ & & - & & $0,39 * * *$ & & $0,51 * * *$ & & - & & $0,53^{* * *}$ \\
\hline Visoesp. & & - & & 0,20 & & $0,24^{*}$ & & - & & $0,32^{* *}$ & & $0,32^{* * *}$ \\
\hline Edad & & 0,03 & & 0,01 & & 0,03 & & 0,01 & & $-0,02$ & & 0,00 \\
\hline
\end{tabular}

Nota. Visoesp. = componente visoespacial; Verbal = componente verbal; Ejecutivo = componente ejecutivo.

${ }^{*} p<0,05 ; * * p<0,01 ; * * * p<0,001$

Modelo 1: Visoesp., $F(3,75)=11,537$

Modelo 2: Verbal, $F(3,75)=8,519$

Modelo 3: Ejecutivo, $F(3,75)=9,301$

Modelo 4: Visoesp., $F(3,78)=23,734$

Modelo 5: Verbal, $F(3,78)=13,284$

Modelo 6: Ejecutivo, $F(3,78)=25,432$

En todos los modelos $p<0,001$

En primer lugar, los resultados obtenidos mostraron que la MT explicó un 33\% de la varianza de la CL. En consonancia con lo hipotetizado, al considerar las contribuciones únicas de cada componente, tanto el ejecutivo como el almacenamiento verbal hicieron aportes específicos significativos, sin que se registrara una contribución significativa del componente de almacenamiento visoespacial. La edad no contribuyó a la explicación de la CL en el modelo de regresión propuesto. La comprensión de los textos expositivo y narrativo presentados requiere que el material leído sea codificado y almacenado verbalmente, en conjunto con recursos ejecutivos para operar sobre esta información y posibilitar la comprensión. En este sentido, los resultados están en línea con la evidencia disponible, que señala que tanto recursos ejecutivos como de almacenamiento están implicados en la CL (Swanson et al., 2009). Si se considera el papel específico de cada componente, el ejecutivo y el almacenamiento verbal contribuyeron de un modo comparable a la explicación de la CL. Tanto la capacidad de retener y mantener activa la información verbal recientemente leída, de recuperar información de la memoria a largo plazo, como de operar con ambas, es decir, integrar el contenido leído previo con el más reciente en una unidad coherente, serían procesos de la MT que están implicados en la CL (Baddeley, 2003b; Cain et al., 2004; Engel de Abreu et al., 2011; Richard's et al., 2014; Swanson et al., 2009). No se observó una contribución significativa de la edad de los participantes a la CL, lo que indica que en niños de 9 a 11 años esta variable no explica una porción de varianza adicional de la CL a la explicada por los componentes de la MT.

Al analizar la contribución explicativa única de cada componente a esta habilidad académica, los resultados muestran que el ejecutivo explica de manera única un 5,9\% de la varianza, el almacenamiento verbal, un 7,6\% y el almacenamiento visoespacial, solo un 1,4\%. El componente ejecutivo de la MT suele ser privilegiado por sobre los demás (Cain et al., 2004; Swanson et al., 2009), por lo que estos resultados resultan de interés, ya que permiten ahondar el conocimiento del rol de cada componente y su peso único en la CL.

En segundo lugar, los resultados obtenidos mostraron que la MT explicó un 52,3\% de la varianza del CM. Si se consideran las contribuciones únicas de cada componente, tanto el ejecutivo como el de almacenamiento verbal y visoespacial hicieron aportes específicos significativos. De la misma manera que en la CL, la edad no contribuyó a la explicación del CM en el modelo de regresión propuesto. Esto indica que, tal como se hipotetizó, todos los componentes de la MT contribuyen conjuntamente a la resolución de operaciones de CM de distinto grado de complejidad, en niños de 9 a 11 años de edad. La resolución de tareas de CM implica la codificación y almacenamiento tanto verbal como visoespacial de información relevante de los problemas aritméticos presentados, así como recursos ejecutivos para operar sobre esta información. Estos resultados son consistentes con la evidencia disponible, que señala que los tres componentes de la MT se relacionan con el desempeño en CM (ver Friso-van den Bos et al., 2013; Raghubar et al., 2010). 
Si se considera el rol específico de cada componente, el almacenamiento verbal es el que contribuyó en mayor medida a esta habilidad académica. Esto puede interpretarse tomando en cuenta la función del bucle fonológico, que en este caso se encargaría de mantener en un estado activo la información numérica relevante a la resolución del problema y los resultados intermedios alcanzados, lo que parece ser un requerimiento central del CM exitoso. En cuanto a los restantes componentes, el ejecutivo mostró un peso relativo menor, aunque explicó una porción importante de la varianza, lo que indica que la resolución de esta clase de problemas implica recursos de procesamiento y manipulación activa de la información. Finalmente, el almacenamiento visoespacial también contribuyó a la explicación del CM, lo que podría estar indicando que la elaboración de una adecuada representación del problema se beneficia de alguna forma de codificación visoespacial del mismo (Bull \& Lee, 2014; Friso-van den Bos et al., 2013; Hecht et al., 2001; Peng et al., 2016; Raghubar et al., 2010). No se observó una contribución significativa de la edad de los participantes al CM, lo que indica que en niños de 9 a 11 años esta variable no contribuye con una porción de varianza adicional a la explicada por los componentes de la MT para predecir el CM.

Al analizar la contribución explicativa única de cada componente a esta habilidad académica, los resultados muestran que el ejecutivo explica de manera única un $2,9 \%$ de la varianza, el almacenamiento verbal un $18,5 \%$, y el almacenamiento visoespacial un $4,6 \%$. Si bien la MT, considerada en conjunto, explica una porción significativa de varianza del CM, es destacable que cada uno de sus componentes explique un porcentaje único, por lo que puede darse cuenta de un rol específico del ejecutivo, del almacenamiento verbal y del visoespacial, cuando son considerados sus pesos relativos en esta habilidad académica. Si solo se considerara a la MT como representada por su aspecto ejecutivo, entonces se perdería el aporte específico de cada componente al CM (Friso-van den Bos et al., 2013; Peng et al., 2016; Raghubar et al., 2010), por lo que este resultado es de interés para aportar mayor evidencia acerca del rol de cada uno de los componentes de la MT en el CM.

En tercer lugar, los resultados muestran que la MT explica una proporción de la varianza mayor en el CM que en la CL. Esto podría suponer que las demandas de la resolución de problemas aritméticos escritos, en términos de almacenamiento y procesamiento, son superiores a las requeridas en la CL, en la cual podrían estar implicadas en mayor medida habilidades verbales y de comprensión específicas (véase Cain et al., 2004).

En síntesis, los resultados de este trabajo muestran que la MT explicaría: (a) una proporción significativa de la CL, a través de sus componentes ejecutivo y de almacenamiento verbal; (b) una proporción significativa del CM, a través de sus componentes ejecutivo, de almacenamiento verbal y visoespacial, y (c) una proporción comparativamente mayor de la varianza del CM que de la varianza de la CL.

En cuanto a las limitaciones del presente estudio, es posible mencionar el tipo de estímulos utilizados en las tareas verbales de MT, dado que estudios previos han registrado que el tipo de estímulo puede afectar el rendimiento y, por ende, la relación con otras variables. Los estímulos de las tareas utilizadas son dígitos y se ha indicado que con este material la relación con la CL puede ser algo menor en comparación con otros tipos de estímulos verbales (Daneman \& Merikle, 1996). Sin embargo, otros autores han señalado que es suficiente contar con tareas que impliquen manipulación de información simbólica (e.g., palabras, oraciones, dígitos) para evaluar adecuadamente los componentes verbales de la MT (Cain et al, 2004). Por otro lado, se ha indicado también que la utilización de dígitos podría llevar a esperar asociaciones más fuertes entre la MT y el CM. No obstante, existe evidencia que muestra que el uso de material numérico en tareas verbales no se ha presentado como un moderador de la relación entre la MT y las diversas habilidades matemáticas a lo largo del ciclo vital (Peng et al., 2016).

Es pertinente considerar, además, que la tarea utilizada para evaluar CM se restringe a la habilidad de resolver problemas aritméticos escritos, lo que claramente no agota la evaluación completa de las diferentes habilidades matemáticas tal como son requeridas en el contexto escolar, como podría ser la resolución de problemas verbales y de geometría, entre otras. Contar con otras pruebas de matemáticas posibilitaría indagar el rol de la MT en diferentes áreas, lo que permitiría esclarecer de manera más completa sus relaciones. Otra de las limitaciones guarda relación con la franja de edad acotada de los participantes (de 9 a 11 años), lo que restringe el alcance de los resultados. Contar con una mayor amplitud de edad permitiría mayor generalización de los resultados.

Se espera que los resultados de este trabajo sean un aporte al conocimiento existente sobre el rol de los diferentes componentes de la MT en las habilidades académicas de CL y CM, pudiendo contribuir, por 
ejemplo, al diseño de programas de intervención destinados a mejorar estas habilidades que tengan en cuenta estas asociaciones. Existen programas de entrenamiento de la MT en niños escolarizados de desarrollo típico que han evaluado la transferencia del entrenamiento al desempeño en matemáticas y lectura, encontrando únicamente transferencia a esta última habilidad (Karbach, Strobach \& Schubert, 2015; Loosli, Buschkuehl, Perrig \& Jaeggi, 2012). Conocer la implicación de cada componente del sistema de MT en la CL y el CM puede permitir la generación de programas de entrenamiento más específicos, que se centren en la estimulación de aquellos procesos que muestran mayor implicación en las habilidades académicas sobre las que se espera impactar.

En conclusión, el presente trabajo reporta evidencia del importante rol de la MT considerada como un sistema y de sus diversos componentes de manera específica en las habilidades académicas de CL y CM en niños argentinos. A la luz de estos resultados, es posible afirmar que la MT no debería ser dejada de lado al estudiar variables asociadas al desempeño académico, ya que constituye un factor clave en estas habilidades de gran importancia en la vida escolar y cotidiana de los niños.

\section{Referencias}

Abusamra, V., Ferreres, A., Raiter, A., De Beni, R. \& Cornoldi, C. (2010). Test Leer Para Comprender. Buenos Aires, Argentina: Paidós.

Alloway, T. P. (2006). How does working memory work in the classroom? Educational Research and Reviews, 1, 134-139.

Alloway, T. P. \& Alloway, R. G. (2010). Investigating the predictive roles of working memory and IQ in academic attainment. Journal of Experimental Child Psychology, 106, 20-29. https://doi.org/10.1016/j.jecp.2009.11.003

Alloway, T. P. \& Copello, E. (2013). Working memory: The what, the why, and the how. The Educational and Developmental Psychologist, 30, 105-118. https://doi.org/10.1017/edp.2013.13

Alloway, T. P., Gathercole, S. E. \& Pickering, S. J. (2006). Verbal and visuospatial short-term and working memory in children: Are they separable? Child Development, 77, 1698-1716. https://doi.org/10.1111/j.1467-8624.2006.00968.x

Alloway, T. P. \& Passolunghi, M. C. (2011). The relationship between working memory, IQ, and mathematical skills in children. Learning and Individual Differences, 21, 133-137. https://doi.org/10.1016/j.lindif.2010.09.013

Alonso, J. \& Mateos, M. M. (1985). Comprensión lectora: modelos, entrenamiento y evaluación. Infancia y Aprendizaje, 8(31-32), 5-19. https://doi.org/10.1080/02103702.1985.10822082

American Psychological Association (2010). Ethical principles of psychologists and code of conduct. Washington, DC: Autor. Extraído de http://www.apa.org/ethics/code/principles.pdf

Baddeley, A. (1992). Working memory. Science, 255, 556-559. https://doi.org/10.1126/science.1736359

Baddeley, A. (1996). Exploring the central executive. The Quarterly Journal of Experimental Psychology Section A, 49, 5-28. https://doi.org/10.1080/713755608

Baddeley, A. (2003a). Working memory: Looking back and looking forward. Nature Reviews: Neuroscience, 4, 829-839. https://doi.org/10.1038/nrn1201

Baddeley, A. (2003b). Working memory and language: An overview. Journal of Communication Disorders, 36, $189-208$. https://doi.org/10.1016/S0021-9924(03)00019-4

Baddeley, A. (2010). Working memory. Current Biology, 20, R136-R140. https://doi.org/10.1016/j.cub.2009.12.014

Baddeley, A. (2012). Working memory: Theories, models and controversies. Annual Review of Psychology, 63, 1-29. https://doi.org/10.1146/annurev-psych-120710-100422

Baddeley, A. D. \& Logie, R. H. (1999). Working memory: The multiple-component model. En A. Miyake \& P. Shah (Eds.), Models of working memory: Mechanisms of active maintenance and executive control (pp. 28-61). New York, NY: Cambridge University Press.

Bennett, K. J., Brown, K. S., Boyle, M., Racine, Y. \& Offord, D. (2003). Does low reading achievement at school entry cause conduct problems? Social Science \& Medicine, 56, 2443-2448. https://doi.org/10.1016/S0277-9536(02)00247-2

Bull, R. \& Lee, K. (2014). Executive functioning and mathematics achievement. Child Development Perspectives, 8, 36-41. https://doi.org/10.1111/cdep.12059

Cain, K., Oakhill, J. \& Bryant, P. (2004). Children's reading comprehension ability: Concurrent prediction by working memory, verbal ability, and component skills. Journal of Educational Psychology, 96, 31-42. https://doi.org/10.1037/0022-0663.96.1.31

Canet-Juric, L., Burin, D., Andrés, M. L. \& Urquijo, S. (2013). Perfil cognitivo de niños con rendimientos bajos en comprensión lectora. Anales de Psicología, 29, 996-1005. https://doi.org/10.6018/analesps.29.3.138221

Canet-Juric, L., Introzzi, I. \& Burin, D. (2015). Desarrollo de la capacidad de memoria de trabajo: efectos de interferencia inter e intra dominio en niños de edad escolar. Revista Argentina de Ciencias del Comportamiento, 7(1), 26-37.

Consejo Nacional de Investigaciones Científicas y Técnicas (2006). Lineamientos para el comportamiento ético en las ciencias sociales y humanidades (CSyH). Buenos Aires, Argentina: Ministerio de Educación, Ciencia y Tecnología. Extraído de http://www.conicet.gov.ar/wp-content/uploads/RD-20061211-2857.pdf

Conway, A. R. A., Kane, M. J., Bunting, M. F., Hambrick, D. Z., Wilhelm, O. \& Engle, R. W. (2005). Working memory span tasks: A methodological review and user's guide. Psychonomic Bulletin \& Review, 12, 769-786. https://doi.org/10.3758/bf03196772

Daneman, M. \& Merikle, P. M. (1996). Working memory and language comprehension: A meta-analysis. Psychonomic Bulletin \& Review, 3, 422-433. https://doi.org/10.3758/BF03214546

Diamond, A. (2012). Activities and programs that improve children's executive functions. Current Directions in Psychological Science, 21, 335-341. https://doi.org/10.1177/0963721412453722

Diamond, A. (2013). Executive functions. Annual Review of Psychology, 64, 135-168. https://doi.org/10.1146/annurev-psych-113011143750 
Engel de Abreu, P. M. J., Gathercole, S. E. \& Martin, R. (2011). Disentangling the relationship between working memory and language: The roles of short-term storage and cognitive control. Learning and Individual Differences, 21, 569-574. https://doi.org/10.1016/j.lindif.2011.06.002

Engle, R. W., Kane, M. J. \& Tuholski, S. W. (1999). Individual differences in working memory capacity and what they tell us about controlled attention, general fluid intelligence, and functions of the prefrontal cortex. En A. Miyake \& P. Shah (Eds.), Models of working memory: Mechanisms of active maintenance and executive control (pp. 102-134). New York, NY: Cambridge University Press.

Friso-van den Bos, I., van der Ven, S. H. G., Kroesbergen, E. H. \& van Luit, J. E. H. (2013). Working memory and mathematics in primary school children: A meta-analysis. Educational Research Review, 10, 29-44. https://doi.org/10.1016/j.edurev.2013.05.003

Gathercole, S. E. (2004). Working memory and learning during the school years. Proceedings of the British Academy, 125, 365-380. https://doi.org/10.5871/bacad/9780197263242.003.0014

Gathercole, S. E., Alloway, T. P., Willis, C. \& Adams, A. -M. (2006). Working memory in children with reading disabilities. Journal of Experimental Child Psychology, 93, 265-281. https://doi.org/10.1016/j.jecp.2005.08.003

Gathercole, S. E., Pickering, S. J., Ambridge, B. \& Wearing, H. (2004). The structure of working memory from 4 to 15 years of age. Developmental Psychology, 40, 177-190. https://doi.org/10.1037/0012-1649.40.2.177

Gathercole, S. E., Pickering, S. J., Knight, C. \& Stegmann, Z. (2004). Working memory skills and educational attainment: Evidence from national curriculum assessments at 7 and 14 years of age. Applied Cognitive Psychology, 18, 1-16. https://doi.org/10.1002/acp.934

Geary, D. C., Hoard, M. K., Nugent, L. \& Byrd-Craven, J. (2008). Development of number line representations in children with mathematical learning disability. Developmental Neuropsychology, 33, 277-299. https://doi.org/10.1080/87565640801982361

Hale, S., Bronik, M. D. \& Fry, A. F. (1997). Verbal and spatial working memory in school-age children: Developmental differences in susceptibility to interference. Developmental Psychology, 33, 364-371. https://doi.org/10.1037/0012-1649.33.2.364

Hale, S., Myerson, J., Emery, L. J., Lawrence, B. M. \& DuFault, C. (2007). Variation in working memory across the life span. En A. R. A. Conway, C. Jarrold, M. J. Kane, A. Miyake \& J. N. Towse (Eds.), Variation in working memory (pp. 194-224). New York, NY: Oxford University Press.

Hecht, S. A., Torgesen, J. K., Wagner, R. K. \& Rashotte, C. A. (2001). The relations between phonological processing abilities and emerging individual differences in mathematical computation skills: A longitudinal study from second to fifth grades. Journal of Experimental Child Psychology, 79, 192-227. https://doi.org/10.1006/jecp.2000.2586

Hofmann, W., Schmeichel, B. J. \& Baddeley, A. D. (2012). Executive functions and self-regulation. Trends in Cognitive Sciences, 16, 174-180. https://doi.org/10.1016/j.tics.2012.01.006

Hollingshead, A. B. (2011). Four factor index of social status. Yale Journal of Sociology, 8, 21-52.

Introzzi, I. M., Canet Juric, L., Comesaña, A., Andrés, M. L. \& Richard's, M. M. (2013). Evaluación de la autorregulación cognitiva y emocional: presentación de un programa. Revista Argentina de Ciencias del Comportamiento, 5(Suplemento Julio: Resúmenes de la XIV Reunión Nacional y III Encuentro Internacional de la Asociación Argentina de Ciencias del Comportamiento), 384-385.

Kane, M. J., Hambrick, D. Z., Tuholski, S. W., Wilhelm, O., Payne, T. W. \& Engle, R. W. (2004). The generality of working memory capacity: A latent-variable approach to verbal and visuospatial memory span and reasoning. Journal of Experimental Psychology: General, 133, 189-217. https://doi.org/10.1037/0096-3445.133.2.189

Karbach, J., Strobach, T. \& Schubert, T. (2015). Adaptive working-memory training benefits reading, but not mathematics in middle childhood. Child Neuropsychology, 21, 285-301. https://doi.org/10.1080/09297049.2014.899336

Loosli, S. V., Buschkuehl, M., Perrig, W. J. \& Jaeggi, S. M. (2012). Working memory training improves reading processes in typically developing children. Child Neuropsychology, 18, 62-78. https://doi.org/10.1080/09297049.2011.575772

Martín Martín, Q., Cabero Morán, M. T. \& de Paz Santana, Y. R. (2008). Tratamiento estadístico de datos con SPSS: prácticas resueltas y comentadas. Madrid, España: Thomson.

McLean, J. F. \& Hitch, G. J. (1999). Working memory impairments in children with specific arithmetic learning difficulties. Journal of Experimental Child Psychology, 74, 240-260. https://doi.org/10.1006/jecp.1999.2516

Miyake, A. \& Shah, P. (Eds.) (1999). Models of working memory: Mechanisms of active maintenance and executive control. New York, NY: Cambridge University Press.

Oakhill, J., Cain, K. \& Elbro, C. (2015). Understanding and teaching reading comprehension: A handbook. New York, NY: Routledge.

Parsons, S. \& Bynner, J. (2005). Does numeracy matter more? London, Reino Unido: National Research and Development Centre for Adult Literacy and Numeracy.

Peng, P., Namkung, J., Barnes, M. \& Sun, C. (2016). A meta-analysis of mathematics and working memory: Moderating effects of working memory domain, type of mathematics skill, and sample characteristics. Journal of Educational Psychology, 108, 455-473. https://doi.org/10.1037/edu0000079

Pickering, S. J. (2001). The development of visuo-spatial working memory. Memory, 9, 423-432. https://doi.org/10.1080/09658210143000182

Pickering, S. J. (Ed.). (2006). Working memory and education. Burlington, MA: Academic Press.

Raghubar, K. P., Barnes, M. A. \& Hecht, S. A. (2010). Working memory and mathematics: A review of developmental, individual difference, and cognitive approaches. Learning and Individual Differences, 20, 110-122. https://doi.org/10.1016/j.lindif.2009.10.005

Richard's, M., Canet-Juric, L., Introzzi, I. \& Urquijo, S. (2014). Intervención diferencial de las funciones ejecutivas en inferencias elaborativas y puente. Avances en Psicología Latinoamericana, 32, 5-20. https://doi.org/10.12804/apl32.1.2014.01

Schmiedek, F., Hildebrandt, A., Lövdén, M., Wilhelm, O. \& Lindenberger, U. (2009). Complex span versus updating tasks of working memory: The gap is not that deep. Journal of Experimental Psychology: Learning, Memory, and Cognition, 35, $1089-1096$. https://doi.org/10.1037/a0015730

Snelbaker, A. J., Wilkinson, G. S., Robertson, G. J. \& Glutting, J. J. (2001). Wide Range Achievement Test 3 (WRAT3). En W. I. Dorfman \& M. Hersen (Eds.), Understanding psychological assessment (pp. 259-274). New York, NY: Springer. https://doi.org/10.1007/978-1-4615-1185-4

St Clair-Thompson, H. L. \& Gathercole, S. E. (2006). Executive functions and achievements in school: Shifting, updating, inhibition, and working memory. The Quarterly Journal of Experimental Psychology, 59, 745-759. https://doi.org/10.1080/17470210500162854 
Swanson, H. L. (2003). Age-related differences in learning disabled and skilled readers' working memory. Journal of Experimental Child Psychology, 85, 1-31. https://doi.org/10.1016/S0022-0965(03)00043-2

Swanson, H. L. \& Alloway, T. P. (2012). Working memory, learning, and academic achievement. En K. R. Harris, S. Graham, T. Urdan, C. B. McCormick, G. M. Sinatra \& J. Sweller (Eds.), APA educational psychology handbook, Vol. 1: Theories, constructs, and critical issues (pp. 327-366). Washington, DC: American Psychological Association. https://doi.org/10.1037/13273-012

Swanson, H. L., Zheng, X. \& Jerman, O. (2009). Working memory, short-term memory, and reading disabilities: A selective metaanalysis of the literature. Journal of Learning Disabilities, 42, 260-287. https://doi.org/10.1177/0022219409331958

Trbovich, P. L. \& LeFevre, J. -A. (2003). Phonological and visual working memory in mental addition. Memory \& Cognition, 31, 738745. https://doi.org/10.3758/BF03196112

Wilkinson, G. S. (1993). WRAT-3: Wide Range Achievement Test administration manual. Wilmington, DE: Wide Range.

Fecha de recepción: Junio de 2016.

Fecha de aceptación: Mayo de 2017. 